\title{
Machine Learning and Fuzzy Logic based Detection, Classification and Grading of Leaf Disease in Plants
}

\author{
Bhavika. N. Patel, Binal kaka, Dweepna Garg, Rima Patel
}

\begin{abstract}
India is an agricultural country where most of people are depends on the agriculture. When Plants are infected by the virus, fungus and bacteria, they are mostly seen on leaves and stems of the plants. Because of that, plants production is decreased also economy of the country is decreased. The farmer has to identify the disease and decide which pesticide will be used to control the disease in plants. To finding out which disease affect the plants, the farmer contacts the expert for the solution. The expert gives the advice based on its knowledge and information but sometimes seeking the expert advice is time consuming, expensive and may be not accurate. So, to solve this problem, the image processing techniques and Machine Learning algorithm like Neural Network, Fuzzy Logic and Support Vector Machine gives the better, accurate and affordable solution to control the plants disease than manual method.
\end{abstract}

Keywords - K Means Clustering; texture feature; Neural Network; GLCM; Leaf

\section{INTRODUCTION}

India is a fast-developing country and agriculture is the backbone for the country's growth [1]. The economic growth is depending on the food production. Plants are considered to be important as they are the source of energy supply to mankind. Plant diseases can affect the leaf any time between scattering and gathering [1]. If plants are infected by bacteria, virus or fungus which can be easily identified from the leaves and stem of the plants, it reduces the production of the food and it directly affects the economical development of the nation. Current world's people has made a massive call for all the food products, so in order to meet the exponential growth of the world's demand; agriculture methods have taken a broad way, by using fertilizers for fast growth. The most important thing is that we have to keep our body healthy. But using these kinds of fertilizers can affect our body. Generally, if we consume any food that is affected by diseases, we obviously get affected by it [3].

Revised Manuscript Received on May 20, 2020.

* Correspondence Author

Bhavika N. Patel*, Computer department, Devang Patel Institute of Advance Technology and Research, Faculty of Technology and Engineering, CHARUSAT, Anand, India, Email: bhavikapatel.dce@ charusat.ac.in

Binal Kaka, Computer department, Devang Patel Institute of Advance Technology and Research, Faculty of Technology and Engineering, CHARUSAT, Anand, India, Email: binalkaka.dce@charusat.ac.in

Dweepna Garg, Computer department, Devang Patel Institute of Advance Technology and Research, Faculty of Technology and Engineering, CHARUSAT, Anand, India, Email: dweepnagarg.ce@charusat.ac.in

Rima Patel, Computer Science \& Engg. department, Devang Patel Institute of Advance Technology and Research, Faculty of Technology and Engineering, CHARUSAT, Anand, India, Email: rimapatel.dcs@charusat.ac.in
To avoid such type of situation, basically farmer contact the expert for the solution. But seeking the expert advice is very time consuming, expensive as it requires continuous monitoring by the experts and inaccurate because they are giving the advice based on their facts and skill. Another issue is, farmer sometimes take the decision by own and use the excessive dose of pesticide, it also affects the food production.

To avoid this entire problem, Machine Learning is a field of computer science, in which a pre-data will be posed to a system and make that system to learn by its own and to apply its learned model, to perform a task [Hari]. Machine learning gives the better solution which is better, accurate and affordable to the farmer than manual method. In our proposed system, first we train our system using training data set then we apply random image of leaf to it and our proposed system will able to identify the disease on leaves and classify according to its category. Also, our proposed system will able to give the grading to the disease means how much disease are affected to the plants.

The plant leaves are affected by bacterial, fungal and viral diseases which include leaf rust, powdery mildew, bacterial blight, Downey mildew, brown spot etc. Figure 1 illustrates the classification of the bacterial, fungal and viral diseases.

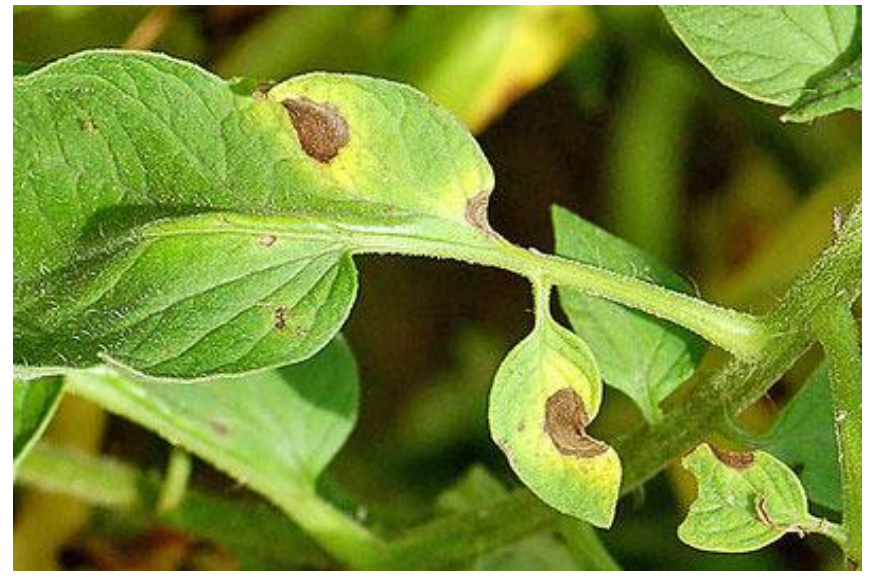

a)Early Bright 


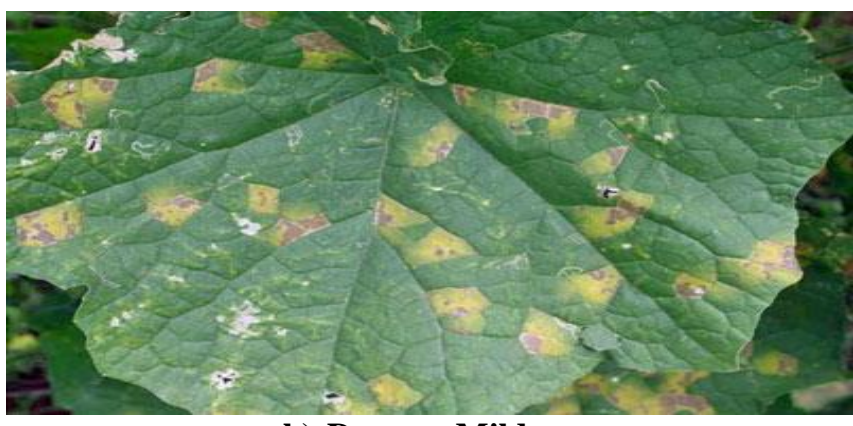

b) Downey Mildew

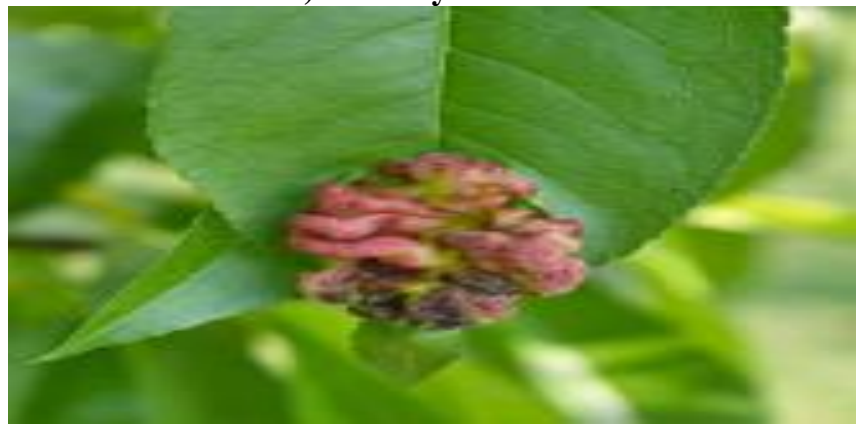

c) Leaf Curl

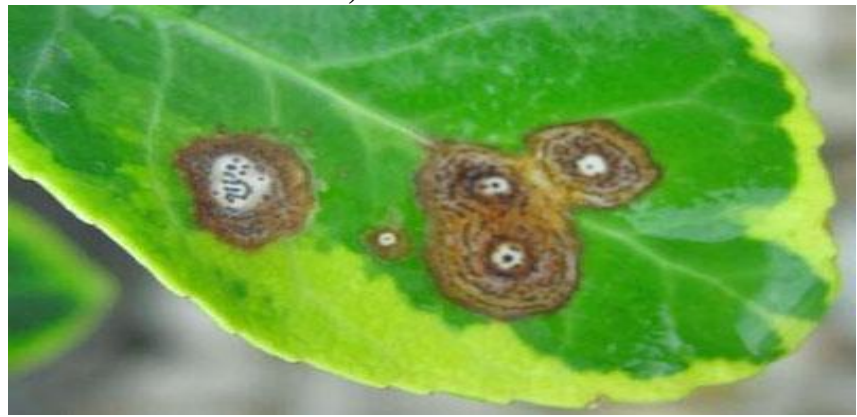

d) Anthracnose

Figure: 1 classification of the bacterial, fungal and viral diseases

\section{LITERATURE SURVEY}

[1] L. sherly Pushpa Annabel, T. Annapoorani and P. Deepalakshmi proposed a "Machine Learning for Plant Leaf Disease Detection and Classification - A Review" which mainly describe the many techniques under Neural Network as supervised and unsupervised learning. And they give the comparative study for different paper based on plants result of their methodology.

[2] Shima Ramesh, Mr. Ramachandra Hebbar, Niveditha M, Pooja R, Prasad Bhat N, Shashank N and Mr. P V Vinod proposed a "Plant Disease Detection Using Machine Learning "which mainly recognize the abnormalities occurs in a plant and they normally concentrate on accuracy. They train their model with 160 images of papaya plants. They used Naïve Bayes, SVM, Logistic Regression and CART for classification. They achieved $70 \%$ accuracy.

[3] S.Santhana Hari, M.Sivakumar, Dr. P.Renuga, S.karthikeyan and S.Suriya proposed a " Detection of Plant Disease by Leaf Image Using Convolutional Neural Network" which mainly implemented on 10 different plants (normal and infected). They developed their model using $\mathrm{CNN}$ which contain different 16 layers. Using this model, they achieved $86 \%$ accuracy.

[4] Siddharth Singh Chouhan, Ajay Kaul, Uday Pratap Singh and Sanjeev Jain has proposed a "Bacterial foraging optimization based Radial Basis Function Neural Network

(BRBFNN) for identification and classification of plant leaf diseases: An automatic approach towards Plant Pathology" mainly focus on fungal disease. They used BRBFNN for detecting and classifying leaf disease in plants.

[5] Juncheng Ma, Keming Du, Feixiang Zheng, Lingxian Zhang, Zhihong Gong and Zhongfu Sun has proposed a "A recognition method for cucumber diseases using leaf symptom images based on deep convolutional neural network" mainly used the Deep Convolutional Neural Network for symptom wise detection for 4 different types of Cucumber disease. They achieved $93.4 \%$ and $92.2 \%$ accuracy for unbalanced dataset and balanced dataset respectively. They also compare their result using AlexaNet.

\section{PROPOSED METHODOLOGY}

The proposed method is developing for automatic detection, classification and grading of leaf diseases in plants as shown in below figure 2 . The dataset of leafs are collected from various sources, it contains basically tomato, cucumber and tobacco leaf images. The dataset contains around 200 images. The proposed system basically divides into 2 parts testing and training. In training module, the leaf images which is infected or normal are given to the proposed method as input and then median and unsharp filter is applied on it for noise remove and sharpening. And then image is segmented into interested region using $\mathrm{K}$ means clustering technique. $\mathrm{K}$ means clustering segmented images into Region of Interest using minimum distance criteria. In K-means clustering, number of clusters are selected randomly or using heuristic approach. Then the minimum distance was calculated from each and every pixel from each center pixel then center pixel was calculated using averaging of every pixel. These steps were following up to no pixel changed its cluster. Each cluster is group of similar pixels whose distance is minimum and different from other clusters pixels. From the all of the cluster, infected cluster was selected and it converted into HSI color image.

When image contain large amount of data, it is necessary to represent data into different format using shape, color and texture. There are many techniques are available for texture feature extraction. Gray Level Co-occurrence matrix (GLCM) is used to extract texture feature from the image. The size of GLCM is depend on number of grey levels in image. GLCM contain the information about how pixel with gray level value $\mathrm{i}$ occurs either vertically $\left(90^{\circ}\right.$ or $\left.270^{\circ}\right)$, horizontally $\left(0^{\circ}\right.$ or $\left.180^{\circ}\right)$ or diagonally (right $\left(45^{\circ}\right.$ or $\left.225^{\circ}\right)$ or left $\left(135^{\circ}\right.$ or $\left.\left.315^{0}\right)\right)$ to its adjacent pixel with gray level value $j$ in image. In this proposed method, the GLCM is calculated for $\mathrm{H}, \mathrm{S}$ and $\mathrm{HS}$ component of image. For this, first RGB image is converted into HSI color space. After generating GLCM, the Haralick's texture features are calculated. Haralick et al. defined 13 texture features like contrast, correlation, energy, entropy, homogeneity etc., for image. Those texture features are used for further classification. These parameters are given to feed forward neural network which is used to train the network. 


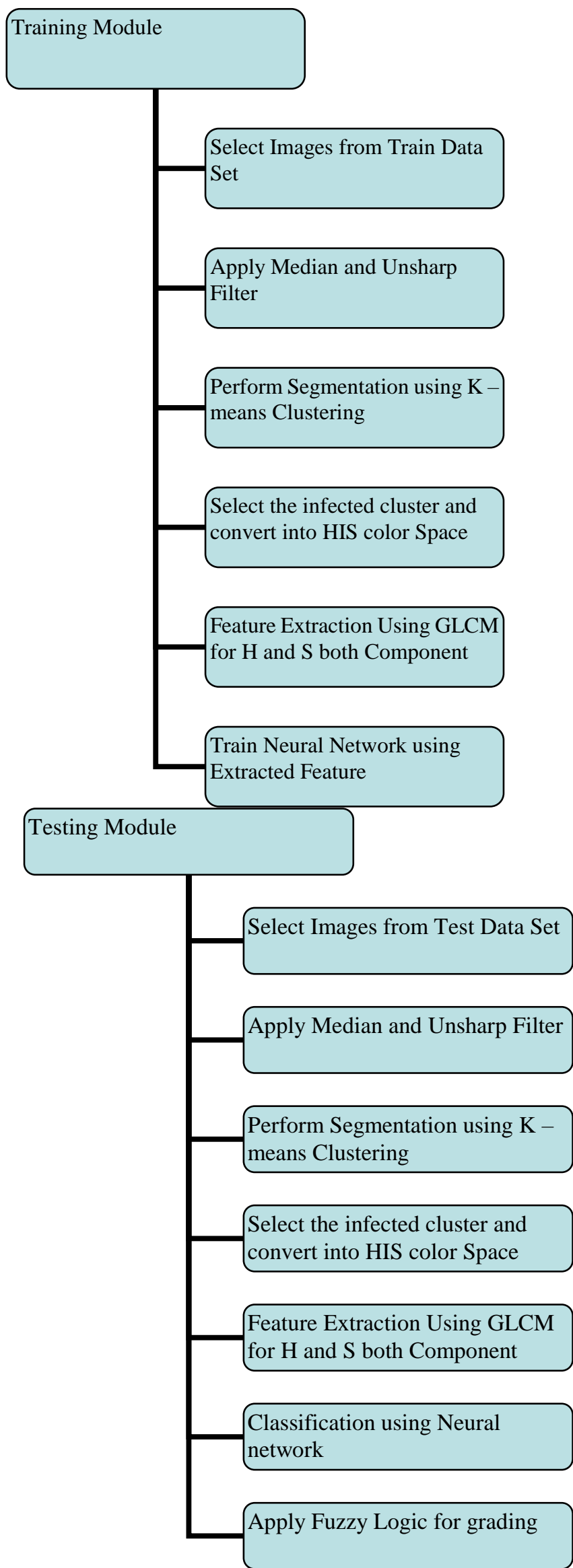

Figure 2 : The Proposed Methodology

In testing module, the testing image is passed through the same steps which are used in training module like first median and unsharp filtering was applied then $\mathrm{K}$ - means clustering is used to segmented the images into different clusters after that texture feature was calculated using GLCM. Then calculated feature are used to identify and classified given image to it belongs disease category. Also, fuzzy logic is used to grade the disease means how much percentages plants are infected. Using this information pesticide are advised.

\section{IMPLEMENTATION RESULTS}

In proposed method, leaf images of anthracnose, leaf curl and powdery mildew disease of different plants are used for detection and classification. Below figures shows some implementation results of proposed method.

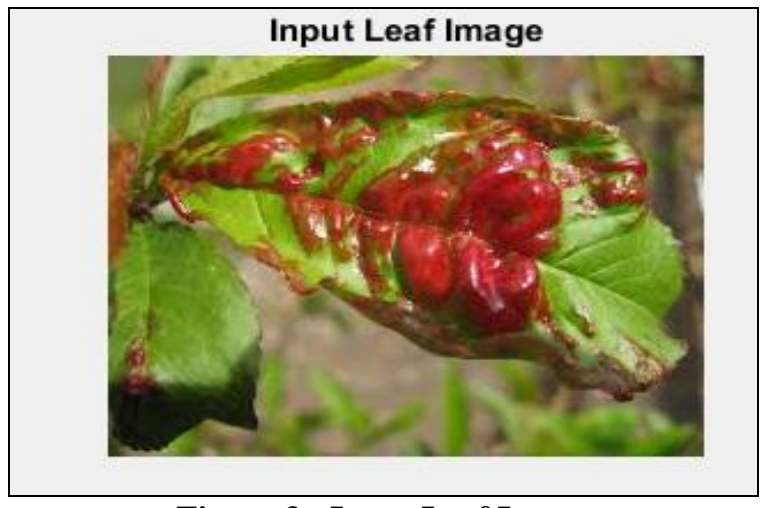

Figure 3 : Input Leaf Image

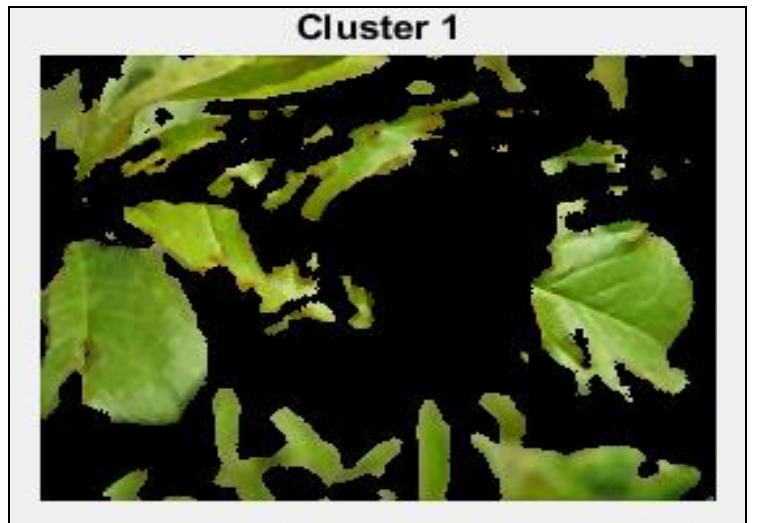

Figure 4 : K means Clustering output (cluster1)

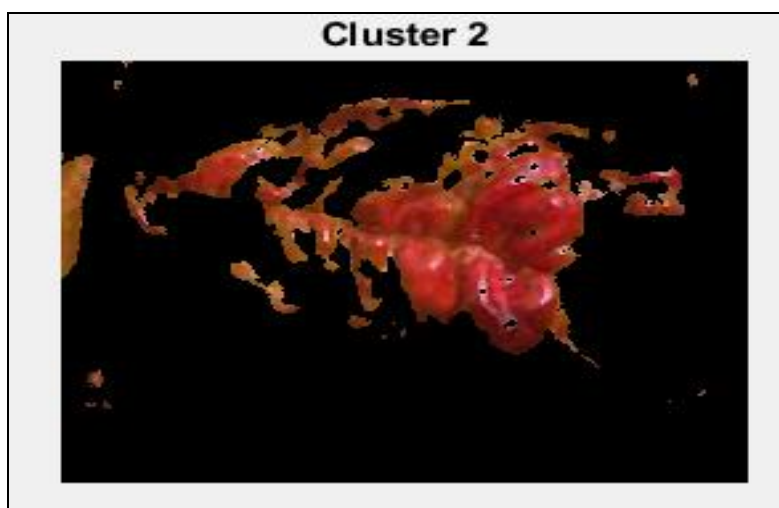

Figure 5 : K means Clustering output (cluster2) 


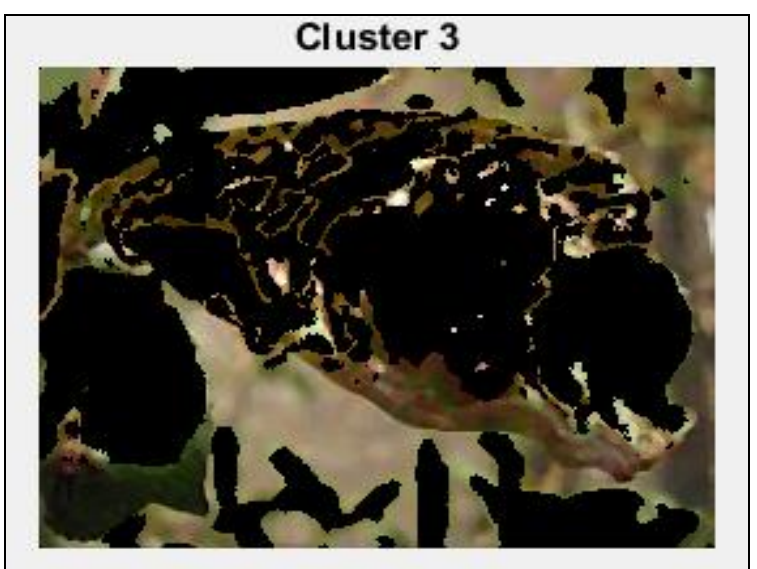

Figure 6 : K means Clustering output (cluster3)

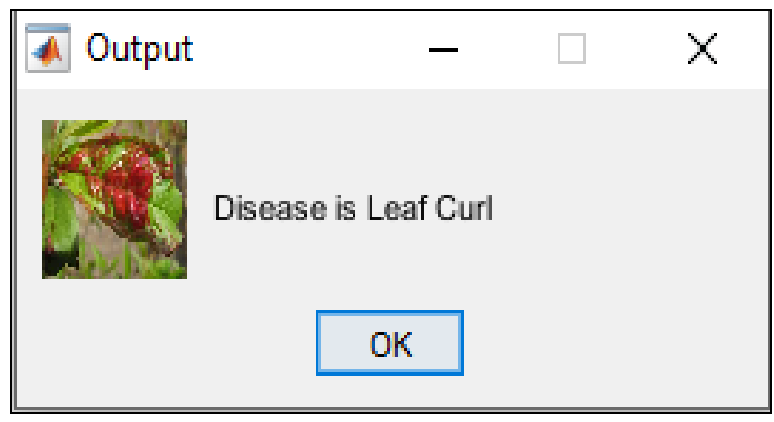

Figure 7 : FFNN output

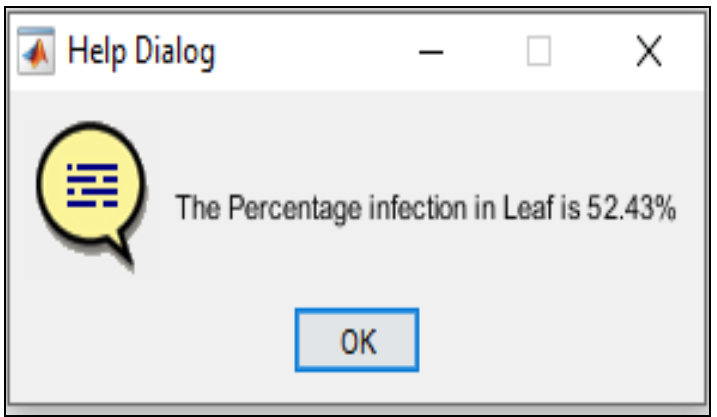

Figure 8 : Fuzzy Logic Output

\begin{tabular}{|c|c|c|c|c|c|c|c|c|}
\hline \multicolumn{9}{|c|}{$\begin{array}{l}\text { > Final_nn } \\
\text { GLCM for H Components : }\end{array}$} \\
\hline 48784 & 0 & 0 & 0 & 0 & 0 & 225 & & \\
\hline 0 & 0 & 0 & 0 & 0 & 0 & 0 & & \\
\hline 0 & 0 & 0 & 0 & 0 & 0 & 0 & & \\
\hline 0 & 0 & 0 & 0 & 0 & 0 & 0 & & \\
\hline 0 & 0 & 0 & 0 & 0 & 0 & 0 & & \\
\hline 0 & 0 & 0 & 0 & 0 & 0 & 0 & & \\
\hline 0 & 0 & 0 & 0 & 0 & 0 & 0 & & \\
\hline 225 & 0 & 0 & 0 & 0 & 0 & 818 & & \\
\hline \multicolumn{9}{|c|}{ Texture Feature for H Component : } \\
\hline Contrast & Correlation & Energy & Homogeneity & Mean & Std_Deviation & Entropy & Variance & IDM \\
\hline 0.440542 & 0.779685 & 0.950282 & 0.992133 & 0.0278373 & -0.14139 & 1.61974 & 0.0174534 & 4.72122 \\
\hline \multicolumn{9}{|c|}{ GLCM for S Components : } \\
\hline 39797 & 0 & 3 & 23 & 116 & 241 & 179 & & \\
\hline 0 & 0 & 0 & 0 & 0 & 0 & 0 & & \\
\hline 6 & 0 & 12 & 10 & 0 & 1 & 0 & & \\
\hline 34 & 0 & 8 & 78 & 18 & 3 & 0 & & \\
\hline 88 & 0 & 8 & 61 & 139 & 31 & 4 & & \\
\hline 151 & 0 & 3 & 19 & 1009 & 349 & 28 & & \\
\hline 255 & 0 & 1 & 3 & 421 & 3612 & 526 & & \\
\hline 147 & 0 & 0 & 0 & 18 & 592 & 1438 & & \\
\hline \multicolumn{9}{|c|}{ Texture Feature for S Component : } \\
\hline Contrast & Correlation & Energy & Homogeneity & Mean & Std_Deviation & Entropy & Variance & IDM \\
\hline 0.931731 & 0.914741 & 0.639164 & 0.952244 & 0.152425 & $\overline{0} .316981$ & 1.99011 & 0.0844042 & 65.7931 \\
\hline
\end{tabular}

Figure 9 : GLCM Features Output

\section{CONCLUSION}

Using Image processing techniques and Machine learning algorithms, the disease in crops are detected, classified and graded, depend on that the solution is advised. The filtering techniques are giving the enhanced result than original image and noise can be removed. This proposed method gives the $82.7 \%$ accurate result and in very short time period. The experimental results show that the proposed approach is a valued methodology, which can give an exact detection of leaf diseases in a little computational effort compare to labor-intensive method.

\section{REFERENCES}

1. L. Sherly Puspha Annabel, Member, IEEE, T. Annapoorani and P. Deepalakshmi, (2019), "Machine Learning for Plant Leaf Disease Detection and Classification - A Review", International Conference on Communication and Signal Processing, April 4-6, 2019, India, IEEE, pp $-0538-0542$.

2. Shima Ramesh, Mr. Ramachandra Hebbar, Niveditha M, Pooja R, Prasad Bhat N, Shashank N and Mr. P V Vinod, (2018), "Plant Disease Detection Using Machine Learning “, International Conference on Design Innovations for 3Cs Compute Communicate Control, DOI 10.1109/ICDI3C.2018.00017, pp- $41-45$.

3. S.Santhana Hari, M.Sivakumar, Dr. P.Renuga, S.karthikeyan and S.Suriya, (2019), “ DETECTION OF PLANT DISEASE BY LEAF IMAGE USING CONVOLUTIONAL NEURAL NETWORK”, International Conference on Vision Towards Emerging Trends in Communication 
and Networking (ViTECoN), pp - $1-5$.

4. Siddharth Singh Chouhan, Ajay Kaul, Uday Pratap Singh and Sanjeev Jain, (2018) "Bacterial foraging optimization based Radial Basis Function Neural Network (BRBFNN) for identification and classification of plant leaf diseases: An automatic approach towards Plant Pathology", IEEE Access, DOI : 10.1109/ACCESS.2017.

5. Juncheng Ma, Keming Du, Feixiang Zheng, Lingxian Zhang, Zhihong Gong and Zhongfu Sun, (2018), "A recognition method for cucumber diseases using leaf symptom images based on deep convolutional neural network", Computers and Electronics in Agriculture, /doi.org/10.1016/j.compag.2018.08.048, pp - 18-24.

6. P. Mohanainah, P. Sathyanarayana and L. Gurukumar, (2013), "Image Texture Feature Extraction using GLCM Approach", International Journal of Scientific and Research Publications, pp. 1-5, vol. 3, Issue 5.

7. Konstantinos G. Liakos, Patrizia Busato, Dimitrios Moshou, Simon Pearson and Dionysis Bochtis, (2018), "Machine Learning in Agriculture: A Review', MDPI Sensors, doi:10.3390/s18082674.

8. Sukhvir Kaur, Shreelekha Pandey and Shivani Goel, (2018), "Plants Disease Identification and Classification Through Leaf Images: A Survey", Archives of Computational Methods in Engineering, Springer, DOI: /10.1007/s11831-018-9255-6.

9. Anandhakrishnan MG Joel Hanson, Annette Joy and Jerin Francis, (2017), "Plant Leaf Disease Detection using Deep Learning and Convolutional Neural Network", International Journal of Engineering Science and Computing.

10. Harshal Waghmare, Yogesh Dandawate, and Radha Kokare (2016), "Detection and Classification of Diseases of Grape Plant Using Opposite Colour Local Binary Pattern Feature and Machine Learning for Automated Decision Support System", 3rd International Conference on Signal Processing and Integrated Networks (SPIN)

\section{AUTHORS PROFILE}

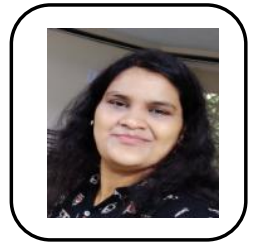

Bbhavika Patel She has received her ME Degree in 2016 from BVM Engg. College. She is currently working as an Assistant Professor in Computer Engg. Dept. at Devanng Patel Institute of Advance Technology and Research, CHARUSAT. Her area of interest are Image Processing and Machine Learning. She has more than 9 years of teaching experience.

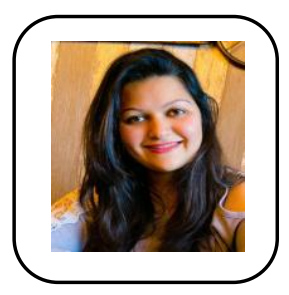

Binal Kaka She is received her ME Degree in 2017 from Parul University. She is currently working as an Assistant Professor in Computer Engg. Dept. at Devanng Patel Institute of Advance Technology and Research, CHARUSAT. Her area of interest are Machine Learning and Data Mining. She has 1.5 years of teaching experience.

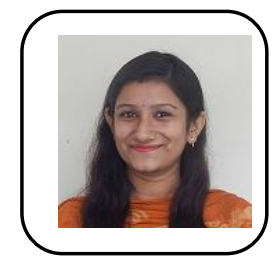

Dweepna Garg She is received her ME Degree in 2015 from Parul University. She is currently served as Head of Computer Engg. Dept. at Devanng Patel Institute of Advance Technology and Research, CHARUSAT. Her area of interest is Machine Learning, Deep Learning and Parrallel Processing. She has more than 7 years of teaching experience.

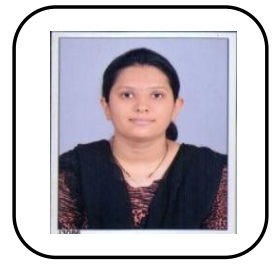

Rima Patel She is received her ME Degree in 2015 from Parul University. She is currently working as an Assistant Professor in Computer Science \& Engg. Dept. at Devanng Patel Institute of Advance Technology and Research, CHARUSAT. Her area of interests is Machine Learning, Data Mining and Block Chain. She has more than 7 years of teaching experience. 